\title{
COMPOSITIONS OF POLYHARMONIC MAPPINGS
}

\author{
GANG LIU AND SAMINATHAN PONNUSAMY
}

\begin{abstract}
The paper is devoted to the study of compositions of polyharmonic mappings in simply connected domains. More precisely, we determine necessary and sufficient conditions of polyharmonic mapping $f$ such that $f \circ F$ (resp. $F \circ f$ ) is $l$-harmonic for any analytic function (or harmonic mapping but not analytic, or $q$-harmonic mapping but not $(q-1)$-harmonic) $F$.
\end{abstract}

\section{Introduction and Main Results}

A complex-valued function $f$ is called a harmonic mapping if it satisfies the harmonic equation $\Delta f=0$, where $\Delta$ denotes the complex Laplacian operator

$$
\Delta=4 \frac{\partial^{2}}{\partial z \partial \bar{z}}=\frac{\partial^{2}}{\partial x^{2}}+\frac{\partial^{2}}{\partial y^{2}} .
$$

Evidently, every harmonic mapping in a simply connected domain admits the representation $f(z)=A(z)+\overline{B(z)}$. Properties of harmonic mappings have been investigated extensively (see the monograph of Duren [15]), especially after the appearance of the pioneering article of Clunie and Sheil-Small [14] in 1984. A simple fact is that a harmonic mapping of an analytic function is harmonic, but an analytic function of a harmonic mapping is not necessarily harmonic. On the other hand, some elementary facts about compositions of certain harmonic mappings, which escape the attention of many, were addressed by Reich [24] in 1987. For instance the following result was established in [24].

Theorem A. Suppose $f(z)=z+\overline{B(z)}$, where $B(z)$ is analytic and $G(z)=B^{\prime}(z)$. $A$ necessary and sufficient condition that there locally exists a non-affine complex harmonic function $g(w)$, such that $g(f(z))$ is harmonic is that $G(z)$ satisfies

$$
\left(G^{\prime}\right)^{2}=\alpha^{2} G^{4}+2 c G^{3}+(\bar{\alpha})^{2} G^{2}
$$

for some complex constant $\alpha$ and some real constant $c$.

In addition to Theorem $A$, several important special cases of it were also obtained in [24] by expressing the analytic functions $G$ and $B$ in terms of elementary functions. The present article is motivated by the work of Reich which provides the local description of all harmonic mappings $f$ such that $g \circ f$ is harmonic for some non-affine harmonic $g$. Twenty years later, properties of the composition of harmonic mappings with harmonic mappings, and the composition of biharmonic mappings with biharmonic mappings were investigated in [7]. Nevertheless, non-analytic and non-harmonic functions play significant

2010 Mathematics Subject Classification. Primary: 31A05, 31A30.

Key words and phrases. analytic, anti-analytic and polyharmonic.

File: LiuSamy1²016 ${ }^{\circ}$.tex, printed: $13-8-2018,3.08$. 
role, eg. biharmonic mappings in fluid dynamics and elasticity problems (see $[16,17,19])$. However, the investigation of biharmonic mappings in the context of geometric function theory is a recent one (see $[1,2,3,6,7,13])$.

We are interested in iterations of the Laplace operator, namely, $p$-harmonic operators defined inductively by $\Delta^{p}=\Delta\left(\Delta^{p-1}\right)$ for $p=2, \ldots$. A $2 p$-times continuously differentiable complex-valued function $f$ in a simply connected domain $D \subseteq \mathbb{C}$ is called $p$-harmonic in $D$ if $f$ satisfies $p$-harmonic equation $\Delta^{p} f=0$ in $D . f$ is called polyharmonic if it is $p$-harmonic for some $p \in \mathbb{N}$. For $p=1$ (resp. 2), $f$ is harmonic (resp. biharmonic). Obviously, every $p$-harmonic mapping is $(p+1)$-harmonic. It is easy to see that, $f$ is $p$-harmonic in a simply connected domain $D$ if and only if (see finite Almansi expansion [4, Proposition 1.3] and [9, Proposition 1.1])

$$
f(z)=\sum_{k=1}^{p}|z|^{2(k-1)} G_{k}(z),
$$

where each $G_{k}(z)$ is harmonic in $D$. There is now a long list of articles in the literature on this subject. For recent results on $p$-harmonic mappings, we refer to the articles $[8,9,10,11,12,18,20,21,22,23]$. Another motivation for the study of polyharmonic mappings is from the recent work of Borichev and Hedenmalm [5] on the study of second order elliptic partial differential equations $T_{\alpha}(f)=0$ in the unit disk $\mathbb{D}=\{z:|z|<1\}$, where $\alpha \in \mathbb{R}$ and

$$
T_{\alpha}=-\frac{\alpha^{2}}{4}\left(1-|z|^{2}\right)^{-\alpha-1}+\frac{\alpha}{2}\left(1-|z|^{2}\right)^{-\alpha-1}\left(z \frac{\partial}{\partial z}+\bar{z} \frac{\partial}{\partial \bar{z}}\right)+\left(1-|z|^{2}\right)^{-\alpha} \frac{\partial^{2}}{\partial z \partial \bar{z}} .
$$

In particular if we take $\alpha=2(p-1)$, then $f$ satisfying $T_{\alpha}(f)=0$ is $p$-harmonic. Clearly, the choice $\alpha=0$ gives that $f$ is harmonic. Moreover, the problem of when the composite mappings of $p$-harmonic mappings with a fixed analytic function are $l$-harmonic was discussed in [21], where $l \in\{1, \ldots, p\}$.

In what follows, the numbers $q, l$ are positive integers unless otherwise stated. $[x]$ denotes the largest integer no more than $x$, where $x$ is a real number. Recall that $f(z)$ is called an affine mapping if $f(z)=\alpha z+\overline{\beta z}+\gamma$, where $\alpha, \beta$ and $\gamma$ are some constants. Similarly, $f$ is called a harmonic polynomial of degree $n$ if $f=h+\bar{g}$, where $h$ and $g$ are analytic polynomials such that $n=\max \{\operatorname{deg} h(z), \operatorname{deg} g(z)\}$.

In this paper we are mainly concerned with the properties of the composition of polyharmonic mappings. We completely solve the following problem: What is the polyharmonic mapping if all its post-compositions by any q-harmonic mapping are l-harmonic? Our main results follow.

Theorem 1. Let $f$ be a polyharmonic mapping. Then

(a) for any analytic $F, f \circ F$ is l-harmonic if and only if $f$ is harmonic;

(b) for any harmonic $F$ which is not analytic, $f \circ F$ is l-harmonic if and only if $f(z)$ is an affine mapping.

(c) for any $q$-harmonic $(q \geq 2) F$ which is not $(q-1)$-harmonic, $f \circ F$ is l-harmonic if and only if $f$ is a harmonic polynomial of degree $t$, where $t \leq \min \left\{1,\left[\frac{l-1}{q-1}\right]\right\}$.

Corollary 1. Let $f$ be an analytic function. Then 
(a) for any harmonic $F$ which is not analytic, $f \circ F$ is l-harmonic if and only if $f(z)$ is linear in $z$.

(b) for any $q$-harmonic $(q \geq 2) F$ which is not $(q-1)$-harmonic, $f \circ F$ is l-harmonic if and only if $f(z)$ is an analytic polynomial of degree $t$, where $t \leq \min \left\{1,\left[\frac{l-1}{q-1}\right]\right\}$.

We next partly solve the problem of characterizing all polyharmonic mappings if all its pre-compositions by any $q$-harmonic mapping are $l$-harmonic.

Theorem 2. Let $f$ be a harmonic mapping. Then

(a) for any harmonic $F, F \circ f$ is l-harmonic if and only if $f$ is analytic or anti-analytic;

(b) for any $q$-harmonic $(q \geq 2) F$ which is not $(q-1)$-harmonic, $F \circ f$ is l-harmonic if and only if either $f(z)$ or $\overline{f(z)}$ is an analytic polynomial of degree $t$, where $t \leq\left[\frac{l-1}{q-1}\right]$

Corollary 2. Let $f$ be an analytic function. Then, for any $q$-harmonic $(q \geq 2) F$ but not $(q-1)$-harmonic, $F \circ f$ is l-harmonic if and only if $f(z)$ is an analytic polynomial of degree $t$, where $t \leq\left[\frac{l-1}{q-1}\right]$.

Theorem 3. Let $f$ be a polyharmonic mapping. Then

(a) for any harmonic $F, F \circ f$ is harmonic (or biharmonic) if and only if $f$ is analytic or anti-analytic;

(b) for any $q$-harmonic $(q \geq 2) F$ which is not $(q-1)$-harmonic, $F \circ f$ is harmonic if and only if $f(z)$ is identically constant;

(c) for any $q$-harmonic $(q \geq 2) F$ which is not $(q-1)$-harmonic, $F \circ f$ is biharmonic if and only if either $f(z)$ or $\overline{f(z)}$ is an analytic polynomial of degree $t$, where $t \leq\left[\frac{1}{q-1}\right]$

The cases where there exist difficulties are formulated as an open problem.

Conjecture 1. Let $f$ be a polyharmonic mapping. Then

(a) for any harmonic $F, F \circ f$ is l-harmonic if and only if $f$ is analytic or anti-analytic;

(b) for any $q$-harmonic $(q \geq 2) F$ which is not $(q-1)$-harmonic, $F \circ f$ is l-harmonic if and only if either $f(z)$ or $\overline{f(z)}$ is an analytic polynomial of degree $t$, where $t \leq\left[\frac{l-1}{q-1}\right]$

\section{SOME NOTATIONS AND PRELIMINARY RESULTS}

For simplicity, we introduce the following notations. For $p \in \mathbb{N}$, we let

$$
\mathbb{H}_{p}=\{f: f \text { is } p \text {-harmonic }\},
$$

and $\mathbb{H}_{0}=\{f: f$ is analytic $\}$. Set $\mathbb{H}_{p}^{*}=\mathbb{H}_{p} \backslash \mathbb{H}_{p-1}$ for $p \geq 1$ and $\mathbb{H}_{0}^{*}=\mathbb{H}_{0}$. Obviously,

$$
\mathbb{H}_{p}=\cup_{i=0}^{p} \mathbb{H}_{i}^{*} \text { and } \mathbb{H}_{i}^{*} \cap \mathbb{H}_{j}^{*}=\emptyset(i \neq j) \text {. }
$$

We observe that if $f$ is $p$-harmonic and is represented by (1) with $G_{p}(z) \neq 0$ for $p \geq 2$, then $f \in \mathbb{H}_{p}^{*}$. Finally, we introduce

$$
\mathbb{H}_{p, q}^{l}=\left\{f: f \in \mathbb{H}_{p} \text { such that } F \circ f \in \mathbb{H}_{l} \text { for any } F \in \mathbb{H}_{q}^{*}\right\},
$$

where $p, q, l$ are non-negative integers. Clearly, constant functions belong to $\mathbb{H}_{p, q}^{l}$. 
Proposition 1. We have the following properties.

(a) $\mathbb{H}_{p, q}^{l} \subseteq \mathbb{H}_{p+1, q}^{l}$ and $\mathbb{H}_{p, q}^{l} \subseteq \mathbb{H}_{p, q}^{l+1}$;

(b) $\mathbb{H}_{p, q}^{l} \subseteq \mathbb{H}_{p, q-1}^{l} \subseteq \cdots \subseteq \mathbb{H}_{p, 1}^{l}=\mathbb{H}_{p, 0}^{l}$;

(c) $\mathbb{H}_{p, q}^{l}=\mathbb{H}_{l, q}^{l}(l \leq p)$.

Proof. (a) The proofs of the inclusions in (a) are trivial.

(b) For the chain of inclusions in (b), we first show that $\mathbb{H}_{p, q+1}^{l} \subseteq \mathbb{H}_{p, q}^{l}$ for any $q \in \mathbb{N}$. Assume that $f \in \mathbb{H}_{p, q+1}^{l}$. Then $f \in \mathbb{H}_{p}$ and $F_{q+1} \circ f \in \mathbb{H}_{l}$ for any $F_{q+1} \in \mathbb{H}_{q+1}^{*}$. Also, for any $F_{q} \in \mathbb{H}_{q}^{*}$, it is easy to see that $F_{q+1}+F_{q} \in \mathbb{H}_{q+1}^{*}$ and

$$
\Delta^{l}\left(F_{q} \circ f\right)=\Delta^{l}\left(\left(F_{q+1}+F_{q}\right) \circ f\right)-\Delta^{l}\left(F_{q+1} \circ f\right)=0 .
$$

Thus, $f \in \mathbb{H}_{p, q}^{l}$ and hence, $\mathbb{H}_{p, q+1}^{l} \subseteq \mathbb{H}_{p, q}^{l}$.

For the completion of the proof of the inclusions in (b), we only need to show that $\mathbb{H}_{p, 1}^{l} \supseteq$ $\mathbb{H}_{p, 0}^{l}$. To do this, we let $F \in \mathbb{H}_{1}^{*}=\mathbb{H}_{1} \backslash \mathbb{H}_{0}$. Then, $F$ is harmonic with the representation $F=h+\bar{g}$, where $h$ and $g$ are analytic, but $g$ is not a constant function. Next, we assume $f \in \mathbb{H}_{p, 0}^{l}$. Then $h \circ f \in \mathbb{H}_{l}$ and $g \circ f \in \mathbb{H}_{l}$. Therefore, $F \circ f=h \circ f+\overline{g \circ f} \in \mathbb{H}_{l}$ and thus, $f \in \mathbb{H}_{p, 1}^{l}$ which shows that $\mathbb{H}_{p, 1}^{l} \supseteq \mathbb{H}_{p, 0}^{l}$.

(c) We claim that $\mathbb{H}_{p, 0}^{l}=\mathbb{H}_{l, 0}^{l}(l \leq p)$. First, we prove that $\mathbb{H}_{p, 0}^{l} \subseteq \mathbb{H}_{l, 0}^{l}$, since $\mathbb{H}_{p, 0}^{l} \supseteq \mathbb{H}_{l, 0}^{l}$ by (a). Next, we assume that $f \in \mathbb{H}_{p, 0}^{l}$. Then $f \in \mathbb{H}_{p}$ and $F \circ f \in \mathbb{H}_{l}$ for any $F \in \mathbb{H}_{0}$. Choosing $F(z)=z$, we see that $f=F \circ f \in \mathbb{H}_{l} \subseteq \mathbb{H}_{p}$. Thus, $f \in \mathbb{H}_{l, 0}^{l}$ and thus, $\mathbb{H}_{p, 0}^{l} \subseteq \mathbb{H}_{l, 0}^{l}$.

Next we show that $\mathbb{H}_{p, q}^{l}=\mathbb{H}_{l, q}^{l}(l \leq p)$. Since $\mathbb{H}_{p, q}^{l} \subseteq \mathbb{H}_{p, 0}^{l}=\mathbb{H}_{l, 0}^{l}$ by (b), it follows that $\mathbb{H}_{p, q}^{l} \subseteq \mathbb{H}_{l, q}^{l}$. Thus, $\mathbb{H}_{p, q}^{l}=\mathbb{H}_{l, q}^{l}$ because of the inclusion $\mathbb{H}_{l, q}^{l} \subseteq \mathbb{H}_{p, q}^{l}$ by (a).

Proposition 2. We have $\mathbb{H}_{2,0}^{2}=\{f: f$ is either analytic or anti-analytic $\}$.

Proof. Set $F_{m}(z)=e^{m z}(m \neq 0)$. Assume that $f \in \mathbb{H}_{2,0}^{2}$. Then $f \in \mathbb{H}_{2}$ and $F_{m} \circ f(z) \in \mathbb{H}_{2}$, which shows that $\Delta^{2}\left(e^{m f}\right)=0$. By computation, we have that

$$
\Delta\left(e^{m f}\right)=4 m e^{m f}\left(f_{z \bar{z}}+m f_{z} f_{\bar{z}}\right) \text { and } \Delta^{2}\left(e^{m f}\right)=16 m^{2} e^{m f} A_{m}(z),
$$

where

$$
A_{m}(z)=2\left(f_{z \bar{z}}^{2}+f_{z} f_{z \bar{z}^{2}}+f_{\bar{z}} f_{z^{2} \bar{z}}\right)+f_{z^{2}} f_{\bar{z}^{2}}+m\left(f_{z}^{2} f_{\bar{z}^{2}}+f_{\bar{z}}^{2} f_{z^{2}}+4 f_{z} f_{\bar{z}} f_{z \bar{z}}\right)+m^{2}\left(f_{z} f_{\bar{z}}\right)^{2} .
$$

Since $e^{m f} \neq 0$ and $m \neq 0$, the equation $\Delta^{2}\left(e^{m f}\right)=0$ is equivalent to $A_{m}(z)=0$. In particular, $A_{2}(z)-A_{1}(z)=0$ and $A_{3}(z)-A_{2}(z)=0$. That is,

$$
\left\{\begin{array}{l}
f_{z}^{2} f_{\bar{z}^{2}}+f_{\bar{z}} f_{z^{2}}+4 f_{z} f_{\bar{z}} f_{z \bar{z}}+3\left(f_{z} f_{\bar{z}}\right)^{2}=0 \\
f_{z}^{2} f_{\bar{z}^{2}}+f_{\bar{z}} f_{z^{2}}+4 f_{z} f_{\bar{z}} f_{z \bar{z}}+5\left(f_{z} f_{\bar{z}}\right)^{2}=0 .
\end{array}\right.
$$

Subtracting the first equation from the second gives $\left(f_{z} f_{\bar{z}}\right)^{2}=0$. Therefore, $f$ is either analytic or anti-analytic. For any analytic function $F, F \circ f$ is then analytic or antianalytic. Obviously, $F \circ f \in \mathbb{H}_{2}$ which implies the desired statement of Proposition 2. 


\section{The PROOFs OF MAIN TheOREMS}

3.1. The proof of Theorem 1. It suffices to prove the necessary parts of the statements (a) to (c), since the sufficiency parts are obvious. Let

$$
f(z)=\sum_{k=1}^{p}|z|^{2(k-1)} G_{k}(z) \neq 0,
$$

where each $G_{k}(z)$ is harmonic.

(a) Assume that $f \circ F \in \mathbb{H}_{l}$ for any analytic function $F$. Let $F(z)=z^{m}(m \in \mathbb{Z}, m>l)$ be given. Then

$$
f \circ F(z)=\sum_{k=1}^{p}|z|^{2 m(k-1)} G_{k}\left(z^{m}\right) \in \mathbb{H}_{l} .
$$

Obviously, each $G_{k}\left(z^{m}\right)$ is still harmonic. Set $t=\max \left\{k: G_{k}(z) \neq 0,1 \leq k \leq p\right\}$. We find that

$$
\sum_{k=1}^{p}|z|^{2 m(k-1)} G_{k}\left(z^{m}\right)=\sum_{k=1}^{t}|z|^{2 m(k-1)} G_{k}\left(z^{m}\right) \in \mathbb{H}_{m(t-1)+1}^{*} .
$$

Thus, by (1), we have that $m(t-1)+1 \leq l$ which implies that $t=1$ and thus, $f(z)=G_{1}(z)$ which is harmonic.

(b) Assume that $f \circ F \in \mathbb{H}_{l}$ for any $F \in \mathbb{H}_{1}^{*}$. Suppose that $F(z)=\bar{z}^{m}(m \in \mathbb{Z}, m>l)$ is given. Then, since for each $k \in\{1, \ldots, p\}, G_{k}\left(\overline{z^{m}}\right)=\overline{G_{k}\left(z^{m}\right)}$ is harmonic, using the similar analysis of the previous case, $f(z)$ must be harmonic. So, we may let

$$
f(z)=\sum_{n=0}^{\infty} \alpha_{n} z^{n}+\overline{\sum_{n=0}^{\infty} \beta_{n} z^{n}}
$$

Again, given $F(z)=z^{m}+(c \bar{z})^{m}(m \in \mathbb{Z}, m>l,|c|=1)$, by a straight calculation, we compute that

$$
\begin{aligned}
\sum_{n=0}^{\infty} \alpha_{n}(F(z))^{n}= & \sum_{n=0}^{\infty} \alpha_{n}\left(\sum_{k=0}^{n} C_{n}^{k} z^{m k}(c \bar{z})^{m(n-k)}\right) \\
= & \sum_{i, j \geq 0} C_{i+j}^{j} \alpha_{i+j} z^{m i}(c \bar{z})^{m j} \\
= & \sum_{n=0}^{\infty} \alpha_{n} z^{m n}+\sum_{n=0}^{\infty} c^{m n} \alpha_{n} \bar{z}^{m n}+\sum_{i, j \geq 1}^{\infty} C_{i+j}^{j} \alpha_{i+j} z^{m i}(c \bar{z})^{m j} \\
= & \sum_{n=0}^{\infty} \alpha_{n} z^{m n}+\sum_{n=0}^{\infty} c^{m n} \alpha_{n} \bar{z}^{m n}+\sum_{j=1}^{\infty} C_{2 j}^{j} c^{m j} \alpha_{2 j}|z|^{2 j m} \\
& +\sum_{i>j \geq 1}^{\infty} C_{i+j}^{j} \alpha_{i+j}\left(c^{m j} z^{m(i-j)}+c^{m i} \bar{z}^{m(i-j)}\right)|z|^{2 j m} \\
= & \sum_{n=0}^{\infty} \alpha_{n} z^{m n}+\sum_{n=0}^{\infty} c^{m n} \alpha_{n} \bar{z}^{m n}+\sum_{j=1}^{\infty} B_{j}(z)|z|^{2 j m}
\end{aligned}
$$


where

$$
B_{j}(z)=C_{2 j}^{j} c^{m j} \alpha_{2 j}+\sum_{i>j}^{\infty} C_{i+j}^{j} \alpha_{i+j}\left(c^{m j} z^{m(i-j)}+c^{m i} \bar{z}^{m(i-j)}\right)
$$

for $j \geq 1$. Similarly, we have

$$
\overline{\sum_{n=0}^{\infty} \beta_{n}(F(z))^{n}}=\sum_{n=0}^{\infty} \bar{\beta}_{n} \bar{z}^{m n}+\sum_{n=0}^{\infty} \bar{c}^{m n} \bar{\beta}_{n} z^{m n}+\sum_{j=1}^{\infty} C_{j}(z)|z|^{2 j m},
$$

where

$$
C_{j}(z)=C_{2 j}^{j} \bar{c}^{m j} \bar{\beta}_{2 j}+\sum_{i>j}^{\infty} C_{i+j}^{j} \bar{\beta}_{i+j}\left(\bar{c}^{m j} \bar{z}^{m(i-j)}+\bar{c}^{m i} z^{m(i-j)}\right)
$$

for $j \geq 1$. Adding the last two expressions, namely, (2) and (3), gives

$$
f \circ F(z)=\sum_{n=0}^{\infty}\left(\alpha_{n}+\bar{c}^{m n} \bar{\beta}_{n}\right) z^{m n}+\sum_{n=0}^{\infty}\left(c^{m n} \alpha_{n}+\bar{\beta}_{n}\right) \bar{z}^{m n}+\sum_{j=1}^{\infty} D_{j}(z)|z|^{2 j m},
$$

where $D_{j}(z)=B_{j}(z)+C_{j}(z)$ for $j \geq 1$, and

$$
\begin{aligned}
D_{j}(z)= & C_{2 j}^{j}\left(c^{m j} \alpha_{2 j}+\bar{c}^{m j} \bar{\beta}_{2 j}\right) \\
& +\sum_{i>j}^{\infty} C_{i+j}^{j}\left(\left(c^{m j} \alpha_{i+j}+\bar{c}^{m i} \bar{\beta}_{i+j}\right) z^{m(i-j)}+\left(c^{m i} \alpha_{i+j}+\bar{c}^{m j} \bar{\beta}_{i+j}\right) \bar{z}^{m(i-j)}\right) .
\end{aligned}
$$

Clearly, each $D_{j}(z)$ is harmonic. Since $f \circ F(z) \in \mathbb{H}_{l}$ and $m>l$, by $(1)$, we have $D_{j}(z) \equiv 0$ for each $j \geq 1$. It can be deduced from Parseval's formula that

$$
c^{m j} \alpha_{2 j}+\bar{c}^{m j} \bar{\beta}_{2 j}=c^{m j} \alpha_{i+j}+\bar{c}^{m i} \bar{\beta}_{i+j}=c^{m i} \alpha_{i+j}+\bar{c}^{m j} \bar{\beta}_{i+j} \equiv 0
$$

for each $i>j \geq 1$ and every $c$ with $|c|=1$. It follows that

$$
\alpha_{2 j}=\beta_{2 j}=\alpha_{i+j}=\beta_{i+j}=0
$$

for all $i>j \geq 1$. Thus, $\alpha_{n}=\beta_{n}=0$ for $n \geq 2$ which yields that

$$
f(z)=\alpha_{0}+\alpha_{1} z+\overline{\beta_{0}+\beta_{1} z} .
$$

(c) Assume that $f \circ F \in \mathbb{H}_{l}$ for any $F \in \mathbb{H}_{q}^{*}(q \geq 2)$.

We first claim that $f$ is a harmonic polynomial and then we show that $f$ is either a constant function or an affine mapping. For this, we begin to consider the representation (1) with

$$
G_{k}(z)=h_{k}(z)+\overline{g_{k}(z)}(1 \leq k \leq p),
$$

where $h_{k}(z)=\sum_{n=0}^{\infty} \alpha_{k, n} z^{n}$ and $g_{k}(z)=\sum_{n=0}^{\infty} \beta_{k, n} z^{n}$. Choosing

$$
F(z)=c|z|^{2(q-1)}(|c|=1)
$$


we find from (1) that

$$
\begin{aligned}
f \circ F(z) & =\sum_{k=1}^{p}|z|^{4(k-1)(q-1)}\left(\sum_{n=0}^{\infty} c^{n} \alpha_{k, n}|z|^{2 n(q-1)}+\overline{\sum_{n=0}^{\infty} c^{n} \beta_{k, n}|z|^{2 n(q-1)}}\right) \\
& =\sum_{k=1}^{p}\left(\sum_{n=0}^{\infty}\left(c^{n} \alpha_{k, n}+\bar{c}^{n} \bar{\beta}_{k, n}\right)|z|^{2(n+2(k-1))(q-1)}\right) \\
& =\sum_{k=1}^{p}\left(\sum_{n=2(k-1)}^{\infty}\left(c^{n-2(k-1)} \alpha_{k, n-2(k-1)}+\bar{c}^{n-2(k-1)} \bar{\beta}_{k, n-2(k-1)}\right)|z|^{2 n(q-1)}\right) \\
& =\sum_{n=0}^{\infty} A_{n}|z|^{2 n(q-1)},
\end{aligned}
$$

where

$$
A_{n}= \begin{cases}\sum_{k=0}^{\left[\frac{n}{2}\right]}\left(c^{n-2 k} \alpha_{k+1, n-2 k}+\bar{c}^{n-2 k} \bar{\beta}_{k+1, n-2 k}\right) & \text { if } n<2(p-1), \\ \sum_{k=0}^{p-1}\left(c^{n-2 k} \alpha_{k+1, n-2 k}+\bar{c}^{n-2 k} \bar{\beta}_{k+1, n-2 k}\right) & \text { if } n \geq 2(p-1) .\end{cases}
$$

Since $f \circ F \in \mathbb{H}_{l}$, by (1), we have $A_{n}=0$ for $n>\left[\frac{l-1}{q-1}\right]$.

Next we deduce that all analytic functions $h_{k}(z)$ and $g_{k}(z)$ should be polynomials. To do this, we fix $n \in \mathbb{N}$ such that

$$
n \geq \max \left\{\left[\frac{l-1}{q-1}\right], 2(p-1)\right\}+1 .
$$

Let $c=w^{i}(i=0,1, \ldots, 2 p-1)$ in $A_{n}=0$, where $w$ is a primitive $4 p n$-th root of unity. Then we have the following equations

$$
\sum_{k=0}^{p-1}\left(\left(w^{i}\right)^{n-2 k} \alpha_{k+1, n-2 k}+\left(\bar{w}^{i}\right)^{n-2 k} \bar{\beta}_{k+1, n-2 k}\right)=0, i=0,1, \ldots, 2 p-1 .
$$

It is easy to see that the coefficient determinant of the above equations is a Vandermonde determinant. As $w$ is a primitive $4 p n$-th root of unity, it is clear that $w^{i} \neq w^{j}(i \neq j)$ and $w^{i} \neq \bar{w}^{j}$ for $i, j \in\{0,1, \ldots, n(2 p-1)\}$. Thus, this Vandermonde determinant does not vanish. It follows that

$$
\alpha_{k+1, n-2 k}=\bar{\beta}_{k+1, n-2 k}=0 \text { for } k=0,1, \ldots, p-1 .
$$

Therefore, all $h_{k}(z)$ and $g_{k}(z)(1 \leq k \leq p)$ are polynomials. If $p=1$, then it is clear that $f$ is a harmonic polynomial. For $p \geq 2$, we will prove that $f$ is also a harmonic polynomial.

Without loss of generality, we may let $h_{k}(z)=\sum_{n=0}^{d} \alpha_{k, n} z^{n}$ and $g_{k}(z)=\sum_{n=0}^{d} \beta_{k, n} z^{n}$ $(d \geq 2,1 \leq k \leq p)$. Again, we choose

$$
F(z)=z^{(m+1)(q-1)} \bar{z}^{q-1}=|z|^{2(q-1)} z^{m(q-1)} \in \mathbb{H}_{q}^{*}(m \in \mathbb{Z}, m>l+d) .
$$


By computation, we have

$$
\begin{aligned}
& f \circ F(z) \\
= & \left.\left.\sum_{k=1}^{p}|| z\right|^{2(q-1)} z^{m(q-1)}\right|^{2(k-1)}\left(\sum_{n=0}^{d} \alpha_{k, n}\left(|z|^{2(q-1)} z^{m(q-1)}\right)^{n}+\overline{\sum_{n=0}^{d} \beta_{k, n}\left(|z|^{2(q-1)} z^{m(q-1)}\right)^{n}}\right) \\
= & \sum_{k=1}^{p} \sum_{n=0}^{d}\left(\alpha_{k, n} z^{n m(q-1)}+\bar{\beta}_{k, n} \bar{z}^{n m(q-1)}\right)|z|^{2\left(t_{k}+n\right)(q-1)} \\
= & \sum_{k=1}^{p} \sum_{n=t_{k}}^{t_{k}+d}\left(\alpha_{k, n-t_{k}} z^{\left(n-t_{k}\right) m(q-1)}+\bar{\beta}_{k, n-t_{k}} \bar{z}^{\left(n-t_{k}\right) m(q-1)}\right)|z|^{2 n(q-1)} \\
= & \sum_{n=0}^{(m+2)(p-1)+d} E_{n}(z)|z|^{2 n(q-1)},
\end{aligned}
$$

where $t_{k}=(m+2)(k-1)$ for $k \in\{1, \ldots, p\}$ and

$$
E_{n}(z)= \begin{cases}\alpha_{k, t} z^{t m(q-1)}+\overline{\beta_{k, t}} \bar{z}^{\operatorname{tm}(q-1)} & \text { for } n=(m+2)(k-1)+t(t \in\{0,1, \ldots, d\}), \\ 0 & \text { otherwise, }\end{cases}
$$

where $k \in\{1, \ldots, p\}$. Obviously, all $E_{n}(z)$ 's are harmonic polynomials. Since $f \circ F(z) \in$ $\mathbb{H}_{l}$ and $(m+2)(k-1)>l$ for $k \in\{2, \ldots, p\}$, by $(1)$, we have $E_{n}(z) \equiv 0$ for $n \geq m+2$. Again, by Parseval's formula, it follows that

$$
\alpha_{k, t}=\beta_{k, t}=0
$$

for $k \in\{2, \ldots, p\}$ and $t \in\{0, \ldots, d\}$. Therefore, $f(z)$ is a harmonic polynomial and thus, for simplicity, we may write it as

$$
f(z)=\sum_{n=0}^{d}\left(\alpha_{n} z^{n}+\overline{\beta_{n} z^{n}}\right)(d>1) .
$$

Now, we show that $f(z)$ is either a constant function or an affine mapping. To do this, we choose $F(z)=c|z|^{2(q-1)}$, where $|c|=1$ and see that

$$
f \circ F(z)=\sum_{n=0}^{d}\left(c^{n} \alpha_{n}+\bar{c}^{n} \bar{\beta}_{n}\right)|z|^{2 n(q-1)} \in \mathbb{H}_{l} .
$$

By the representation (1), we conclude that

$$
c^{n} \alpha_{n}+\bar{c}^{n} \bar{\beta}_{n}=0
$$

for any $n>\frac{l-1}{q-1} \geq\left[\frac{l-1}{q-1}\right]=t$ and $c$ with $|c|=1$. This gives $\alpha_{n}=\beta_{n}=0$ for $n>t$. Next we divide the proof into three cases.

(i) If $t=0$, then $l<q$ and thus, $f(z)=\alpha_{0}+\overline{\beta_{0}}$ which is a constant. Obviously, $f \circ F(z)$ reduces to a constant and hence, belongs to $\in \mathbb{H}_{l}$ for any $F(z) \in \mathbb{H}_{q}^{*}$.

(ii) If $t=1$, then $q \leq l \leq 2 q-2$ and $f(z)=\alpha_{0}+\alpha_{1} z+\overline{\beta_{0}+\beta_{1} z}$. Obviously, $f \circ F(z) \in \mathbb{H}_{q} \subset \mathbb{H}_{l}$ for any $F(z) \in \mathbb{H}_{q}^{*}$. 
(iii) If $t \geq 2$, then $l>2 q-2$. We claim that $\alpha_{n}=\beta_{n}=0$ for $2 \leq n \leq t$. Again, let

$$
F(z)=c|z|^{2(q-1)}\left(z^{2 m}+\bar{z}^{2 m}\right)(m \in \mathbb{Z}, m>l,|c|=1) .
$$

Then, again by (1), we have

$$
\begin{aligned}
f \circ F(z)= & \sum_{n=0}^{1}\left(c^{n} \alpha_{n}+\bar{c}^{n} \bar{\beta}_{n}\right)|z|^{2 n(q-1)}\left(z^{2 m}+\bar{z}^{2 m}\right)^{n} \\
& +\sum_{n=2}^{t}\left(c^{n} \alpha_{n}+\bar{c}^{n} \bar{\beta}_{n}\right)|z|^{2 n(q-1)}\left(z^{2 m}+\bar{z}^{2 m}\right)^{n} \\
= & H(z)+\sum_{n=2}^{t}\left(c^{n} \alpha_{n}+\bar{c}^{n} \bar{\beta}_{n}\right) H_{n}(z),
\end{aligned}
$$

where

$$
H(z)=\sum_{n=0}^{1}\left(c^{n} \alpha_{n}+\bar{c}^{n} \bar{\beta}_{n}\right)|z|^{2 n(q-1)}\left(z^{2 m}+\bar{z}^{2 m}\right)^{n} \text { and } H_{n}(z)=|z|^{2 n(q-1)}\left(z^{2 m}+\bar{z}^{2 m}\right)^{n} .
$$

Clearly, $H(z) \in \mathbb{H}_{q}$. However, if $n \geq 2$ is an even number, then one writes

$$
\begin{aligned}
H_{n}(z) & =|z|^{2 n(q-1)} \sum_{k=0}^{n} C_{n}^{k} z^{2 m(n-k)} \bar{z}^{2 m k} \\
& =|z|^{2 n(q-1)}\left(C_{n}^{\frac{n}{2}}|z|^{2 n m}+\sum_{k=0}^{\frac{n}{2}-1}\left(C_{n}^{k} z^{2 m(n-2 k)}|z|^{4 m k}+C_{n}^{\frac{n}{2}+k+1} \bar{z}^{4 m(k+1)}|z|^{2 m(n-2-2 k)}\right)\right),
\end{aligned}
$$

and thus, it follows from $(1)$ that $H_{n}(z) \in \mathbb{H}_{n(m+q-1)+1}^{*}$ and $H_{n}(z)$ can be expressed as

$$
H_{n}(z)=C_{n}^{\frac{n}{2}}|z|^{2 n(m+q-1)}+\widetilde{H_{n}}(z)
$$

where $\widetilde{H_{n}}(z) \in \mathbb{H}_{n(m+q-1)}$. If $n \geq 2$ is an odd number, then it is easy to deduce that $H_{n}(z) \in \mathbb{H}_{n(m+q-1)-m+1}^{*}$ and

$$
H_{n}(z)=C_{n}^{\frac{n-1}{2}}\left(z^{2 m}+\bar{z}^{2 m}\right)|z|^{2(n(m+q-1)-m)}+\widehat{H_{n}}(z),
$$

where $\widehat{H_{n}}(z) \in \mathbb{H}_{n(m+q-1)-m}$. Note that both $n(m+q-1)+1$ and $n(m+q-1)-m+1$ are strictly monotonically increasing as $n$ from 2 to $t$. Moreover, there are no integers $n_{1}, n_{2} \in[2, t]$ such that $n_{1}(m+q-1)+1=n_{2}(m+q-1)-m+1$. Therefore, if $t$ is an even number, then $f \circ F$ has the following form

$$
f \circ F(z)=C_{t}^{\frac{t}{2}}\left(c^{t} \alpha_{t}+\bar{c}^{t} \bar{\beta}_{t}\right)|z|^{2 t(m+q-1)}+L_{t}(z),
$$

where $L_{t}(z) \in \mathbb{H}_{t(m+q+1)}$. Since $f \circ F \in \mathbb{H}_{l}$ and $t(m+q-1)+1>l+1$, by (1), we get

$$
c^{t} \alpha_{t}+\bar{c}^{t} \bar{\beta}_{t}=0
$$

for any $c$ with modulus one. Thus, $\alpha_{t}=\beta_{t}=0$. If $t>2$, then $t-1$ is a odd number. Therefore, $f \circ F$ can be written as

$$
f \circ F=C_{t-1}^{\frac{t-2}{2}}\left(c^{t-1} \alpha_{t-1}+\bar{c}^{t-1} \bar{\beta}_{t-1}\right)\left(z^{2 m}+\bar{z}^{2 m}\right)|z|^{2((t-1)(m+q-1)-m)}+L_{t-1}(z),
$$


where $L_{t-1}(z) \in \mathbb{H}_{(t-1)(m+q-1)-m}$. Since $f \circ F \in \mathbb{H}_{l}$ and $(t-1)(m+q+1)-m>l+1$, by (1), we have that

$$
c^{t-1} \alpha_{t-1}+\bar{c}^{t-1} \bar{\beta}_{t-1}=0,
$$

for any $c$ with modulus one. Thus, $\alpha_{t-1}=\beta_{t-1}=0$. If $t=3$, the proof is finished. If $t>3$, we can similarly obtain that

$$
\alpha_{t-2}=\beta_{t-2}=\cdots=\alpha_{2}=\beta_{2}=0,
$$

since $n(m+q-1)+1$ and $n(m+q-1)-m+1$ are greater than $l+1$ for any $n \in\{2, \ldots, t\}$.

If $t$ is an odd number, then, by a similar analysis in the even case, we get that

$$
\alpha_{t}=\beta_{t}=\cdots=\alpha_{2}=\beta_{2}=0 .
$$

In other words, if $f \circ F \in \mathbb{H}_{l}$ for any $F \in \mathbb{H}_{q}^{*}(q \geq 2)$, then $f(z)=\sum_{n=0}^{t} \alpha_{n} z^{n}+\overline{\sum_{n=0}^{t} \beta_{n} z^{n}}$, where $t \leq \min \left\{1,\left[\frac{l-1}{q-1}\right]\right\}$. The proof of Theorem 1 is finished.

3.2. The proof of Theorem 2. The statement (a) is equivalent to proving

$$
\mathbb{H}_{1,0}^{l} \cap \mathbb{H}_{1,1}^{l}=\{f: f \text { is either analytic or anti-analytic }\} \text {. }
$$

By Proposition 1, we have $\mathbb{H}_{1,1}^{l}=\mathbb{H}_{1,0}^{l}$ and thus, it suffices to prove

$$
\mathbb{H}_{1,0}^{l}=\{f: f \text { is either analytic or anti-analytic }\} \text {. }
$$

Let $f \in \mathbb{H}_{1,0}^{l}$. Then, $f(z)$ is harmonic and thus, has the form $f(z)=h(z)+\overline{g(z)}$, where $h(z)$ and $g(z)$ are analytic. Set

$$
F(z)=\left(e^{z}\right)^{m}(m \in \mathbb{Z}, m>l) .
$$

Then we get that

$$
F \circ f(z)=\left(e^{h(z)} \overline{e^{g(z)}}\right)^{m}=H^{m}(z) \overline{G^{m}(z)},
$$

where $H(z)=e^{h(z)}$ and $G(z)=e^{g(z)}$ are analytic. Since $F \circ f \in \mathbb{H}_{l}$, we see that

$$
\Delta^{l}(F \circ f(z))=\Delta^{l}\left(H^{m}(z) \overline{G^{m}(z)}\right)=4^{l} \frac{\partial^{l} H^{m}(z)}{\partial z^{l}} \frac{\partial^{l} \overline{G^{m}(z)}}{\partial \bar{z}^{l}}=0,
$$

which yields

$$
\text { either } \frac{\partial^{l} H^{m}(z)}{\partial z^{l}}=0 \text { or } \frac{\partial^{l} G^{m}(z)}{\partial z^{l}}=0 .
$$

Thus, either $H^{m}(z)$ or $G^{m}(z)$ is a polynomial with degree not more than $l-1$. Note that $m>l$. Consequently, either $H(z)$ or $G(z)$ is a constant which in turn implies that either $h(z)$ or $g(z)$ is a constant. Therefore, $f(z)$ is analytic or anti-analytic. Obviously, for any harmonic $F$ and any analytic or anti-analytic $f, F \circ f$ is harmonic which is also l-harmonic.

(b) The statement (b) is equivalent to showing that

$$
\mathbb{H}_{1, q}^{l}=\left\{f: f=\sum_{n=0}^{t} \alpha_{n} z^{n} \quad \text { or } f(z)=\overline{\sum_{n=0}^{t} \beta_{n} z^{n}}\right\},
$$

where $q \geq 2$ and $t \leq\left[\frac{l-1}{q-1}\right]$. By Proposition 1 , we have $\mathbb{H}_{1, q}^{l} \subseteq \mathbb{H}_{1,0}^{l}$. Thus, if $f \in \mathbb{H}_{1, q}^{l}$, then either $f$ is analytic or anti-analytic. 
Since $F \circ \bar{f}=\overline{\bar{F} \circ f}$, we only need to consider the case that $f$ is analytic. Let $F(z)=$ $|z|^{2(q-1)}\left(\in \mathbb{H}_{q}^{*}\right)$. Then we have that

$$
H(z)=F \circ f(z)=f^{q-1}(z) \bar{f}^{q-1}(z) .
$$

If $H \in \mathbb{H}_{l}$, then we have

$$
\Delta^{l} H(z)=4^{l} \frac{\partial^{l} f^{q-1}(z)}{\partial z^{l}} \frac{\partial^{l} \bar{f}^{q-1}(z)}{\partial \bar{z}^{l}}=0,
$$

which yields $\frac{\partial^{l} f^{q-1}(z)}{\partial z^{l}}=0$. Therefore, $f^{q-1}(z)=\sum_{n=0}^{l-1} \beta_{n} z^{n}$ which implies that $f$ must be a polynomial of degree not more than $\left[\frac{l-1}{q-1}\right]$ and thus, we write $f(z)=\sum_{n=0}^{t} \alpha_{n} z^{n}\left(t \leq\left[\frac{l-1}{q-1}\right]\right)$. For any $F \in \mathbb{H}_{q}^{*}(q \geq 2), F$ has the representation

$$
F(z)=\sum_{k=1}^{q}|z|^{2(k-1)} G_{k}(z)
$$

where each $G_{k}(z)$ is harmonic and $G_{q}(z) \neq 0$. By computation, we may then write

$$
\begin{aligned}
F \circ f(z) & =\sum_{k=1}^{q}\left(\sum_{n=0}^{t} \alpha_{n} z^{n}\right)^{k-1} \overline{\left(\sum_{n=0}^{t} \alpha_{n} z^{n}\right)^{k-1}} G_{k}(f(z)) \\
& =\sum_{k=1}^{q}\left(\sum_{n=0}^{t(k-1)} c_{n} z^{n}\right)\left(\sum_{n=0}^{t(k-1)} \overline{c_{n}} \bar{z}^{n}\right) G_{k}(f(z)) \text { for some } c_{n} \text { 's } \\
& =\sum_{k=1}^{q}\left(\sum_{0 \leq i, j \leq t(k-1)} C_{i j} z^{i} \bar{z}^{j} G_{k}(f(z))\right)
\end{aligned}
$$

where each $C_{i, j}$ is a complex number. It is easy to deduce that $z^{i} \bar{z}^{j} G_{k}(f(z))$ is $\max \{i+$ $1, j+1$-harmonic. Since $i, j \leq t(q-1)$, we see that $F \circ f \in \mathbb{H}_{t(q-1)+1}$. As $t(q-1)+1 \leq l$, it is obvious that $F \circ f \in \mathbb{H}_{l}$. The proof is complete.

3.3. The proof of Theorem 3. The sufficiency parts of the statements in (a)-(c) are obvious and therefore, we need to prove only the necessary part of the theorem.

(a) The statement (a) is equivalent to proving

$$
\mathbb{H}_{p, 0}^{1} \cap \mathbb{H}_{p, 1}^{1}=\mathbb{H}_{p, 0}^{2} \cap \mathbb{H}_{p, 1}^{2}=\{f: f \text { is either analytic or anti-analytic }\},
$$

where $p \geq 2$. By Proposition 1 and Theorem 2, we acquire

$$
\mathbb{H}_{p, 1}^{1}=\mathbb{H}_{p, 0}^{1}=\mathbb{H}_{1,0}^{1}=\{f: f \text { is either analytic or anti-analytic }\} \text {. }
$$

By Propositions 1 and 2, we get

$$
\mathbb{H}_{p, 1}^{2}=\mathbb{H}_{p, 0}^{2}=\mathbb{H}_{2,0}^{2}=\{f: f \text { is either analytic or anti-analytic }\} \text {. }
$$

(b) By Proposition 1, we have $\mathbb{H}_{p, q}^{1}=\mathbb{H}_{1, q}^{1}$. Therefore, the statement is an immediate consequence of Theorem 2.

(c) By Proposition 1, we obtain that $\mathbb{H}_{p, q}^{2}=\mathbb{H}_{2, q}^{2} \subseteq \mathbb{H}_{2,0}^{2}$. By Proposition 2, it follows that $\mathbb{H}_{p, q}^{2} \subseteq \mathbb{H}_{1, q}^{2}$ and the desired conclusion follows. 
Acknowledgments. The research was supported by the NSFs of China (No.11371363), the construct program of the key discipline in Hunan province and the Hunan Provincial Natural Science Foundation of China (No.2015JJ6011).

\section{REFERENCES}

[1] Z. Abdulhadi and Y. Abu Muhanna, Landau's theorem for biharmonic mappings, J. Math. Anal. Appl., 338 (2008), 705-709.

[2] Z. Abdulhadi, Y. Abu Muhanna and S. Khuri, On univalent solutions of the biharmonic equation, J. Inequal. Appl., 5 (2005), 469-478.

[3] Z. Abdulhadi, Y. Abu Muhanna and S. Khoury, On some properties of solutions of the biharmonic equation, Appl. Math. Comput., 177 (2006), 346-351.

[4] N. Aronszajn, T.M. Creese and L.J. Lipkin, Polyharmonic functions, Clarendon Press, Oxford, 1983.

[5] A. Borichev and H. Hedenmalm, Weighted integrability of polyharmonic functions, Adv. Math., 264(2014), 464-505.

[6] SH. Chen, S. Ponnusamy and X. Wang, Landau's theorem for certain biharmonic mappings, Appl. Math. Comput., 208 (2009), 427-433.

[7] SH. Chen, S. Ponnusamy and X. Wang, Compostions of harmonic mappings and biharmonic mappings, Bull. Belg. Math. Soc. Simon Stein, 17(2010), 693-704.

[8] SH. Chen, S. Ponnusamy and X. Wang, Bloch and Landau's theorems for planar p-harmonic mappings, J. Math. Anal. Appl., 373 (2011), 102-110.

[9] SH. Chen, S. Ponnusamy and X. Wang, On some properties of solutions of the p-harmonic equations, Filomat, 27 (2013), 577-591.

[10] J. Chen, A. Rasila and X. Wang, On polyharmonic univalent mappings, Math. Rep., 15 (2013), $343-357$.

[11] J. Chen, A. Rasila and X. Wang, Starlikeness and convexity of polyharmonic mappings, Bull. Belg. Math. Soc. Simon Stevin., 21 (2014), 67-82.

[12] J. Chen, A. Rasila and X. Wang, Landau's theorem for polyharmonic mappings, J. Math. Anal. Appl., 409 (2014), 934-945.

[13] J. Chen and X. Wang, On certain classes of biharmonic mappings defined by convolution, Abstr. Appl. Anal., 2012, Article ID 379130, 10 pages. doi:10.1155/2012/379130.

[14] J. G. Clunie and T. Sheil-Small, Harmonic univalent functions, Ann. Acad. Sci. Fenn. Ser. A. I. 9 (1984), 3-25.

[15] P. Duren, Harmonic mappings in the plane, Cambridge University Press, Cambridge, 2004.

[16] J. Happel and H. Brenner, Low Reynolds Number Hydrodynamics, Princeton-Hall, 1965.

[17] S. A. Khuri, Biorthogonal series solution of Stokes flow problems in sectorial regions, SIAM J. Appl. Math., 56 (1996), 19-39.

[18] S. A. Khuri, On the properties of a class of polyharmonic functions, Math. Model. Anal., 18 (2013), 219-235.

[19] W. E. Langlois, Slow Viscous Flow, Macmillan Company, 1964.

[20] P. Li, S. A. Khuri, X. Wang, On certain geometric properties of polyharmonic mappings, J. Math. Anal. Appl., 434 (2016), 1462-1473.

[21] P. Li, S. Ponnusamy and X. Wang, Some properties of planar p-harmonic and log-p-harmonic mappings, Bull. Malaysian Math. Sciences Soc., 36 (2013), 595-609.

[22] J. Qiao and X. Wang, Subordination of p-harmonic mappings, Bull. Belg. Math. Soc. Simon Stevin, 19 (2012), 47-61.

[23] J. Qiao and X. Wang, On p-harmonic univalent mappings (in Chinese), Acta Math. Sci., 32 (2012), 588-600.

[24] E. Reich, The composition of harmonic mappings, Ann. Acad. Sci. Fenn. Ser. A. I, 12 (1987), $47-53$. 
Gang Liu, College of Mathematics and Statistics (Hunan Provincial Key Laboratory of Intelligent Information Processing and Application), Hengyang Normal University, Hengyang, Hunan 421008, People's Republic of China.

E-mail address: liugangmath@sina.cn

Saminathan Ponnusamy, Indian Statistical Institute (ISI), Chennai Centre, SETS (Society for Electronic Transactions and Security), MGR Knowledge City, Cit Campus, Taramani, Chennai 600 113, India.

E-mail address: samy@isichennai.res.in, samy@iitm.ac.in 